\title{
Three-dimensional microanalysis using FIB SEM: Variations in technique
}

\author{
Keana Scott*, Jeffrey M. Davis*, and Edward P. Vicenzi** \\ *National Institute of Standards and Technology, 100 Bureau Drive, Gaithersburg, MD \\ 20899 \\ ** Smithsonian Institution, Museum Conservation Institute, Suitland, MD 20746
}

In recent years, by combining energy dispersive x-ray spectrometry (EDS) and automated three-dimensional (3D) imaging using focused ion beam scanning electron microscopy (FIB SEM), multiple groups have demonstrated 3D microanalysis of complex microstructures $[1,2]$. In addition to the usual analytical challenges associated with traditional microanalysis methods, the FIB-based 3D method comes with several additional complications. In this method, the analysis surface is prepared by removing material surrounding the feature of interest to permit access by the electron beam and to create an unobstructed view for the x-ray detector. In many cases spurious contributions to the analytical signals can arise from the base and the walls of the pit surrounding the analysis volume, complicating reliable quantitative analysis. In their recent paper, Schaffer et al. suggest lifting the volume of interest from the bulk sample and performing the EDS analysis with the volume suspended in vacuum as a possible alternative [3]. The non-optimal takeoff angle of the EDS detector in some instruments is another possible complication [4]. Performing additional stage movements and/or rotations following each milling step can mitigate the takeoff angle problem in some cases, but at the expense of increased data acquisition time.

In our study, we examined several different lift out methods to minimize the contributions from the surrounding material and to improve the EDS detector takeoff angle. Using homogeneous NIST reference raw materials, we fabricated bulk samples containing micro-inclusions with known composition. Compositional analysis results from various experimental conditions are compared and errors associated with $3 \mathrm{D}$ compositional analysis using the FIB-based technique are evaluated. Roughly $10 \mu \mathrm{m}$ x 10 $\mu \mathrm{m} \times 10 \mu \mathrm{m}$ of sample material was lifted out for each experimental condition. By varying the rotation and tilt of the stage before performing the lift out operation and by adjusting the rotation of the sequential FIB milling pattern to match the analysis surface, we can vary the takeoff angle of the analysis surface. We also evaluated the contribution from the surrounding material by performing FIB milling and EDS spectrum imaging with the sample suspended in vacuum (Fig. 1) and compared the results to matched experiments using a clean substrate such as a carbon planchet (Fig. 2).

\section{References}

[1] P. Kotula et al., Microsc. Microanal. 12 (2006) 36.

[2] M. Uchic et al., MRS Bulletin 32 (2007) 408.

[3] M. Schaffer et al., Microsc. Microanal. 14(Suppl 2) (2008) 982.

[4] M. Schaffer et al., Ultramicroscopy 107 (2007) 587. 

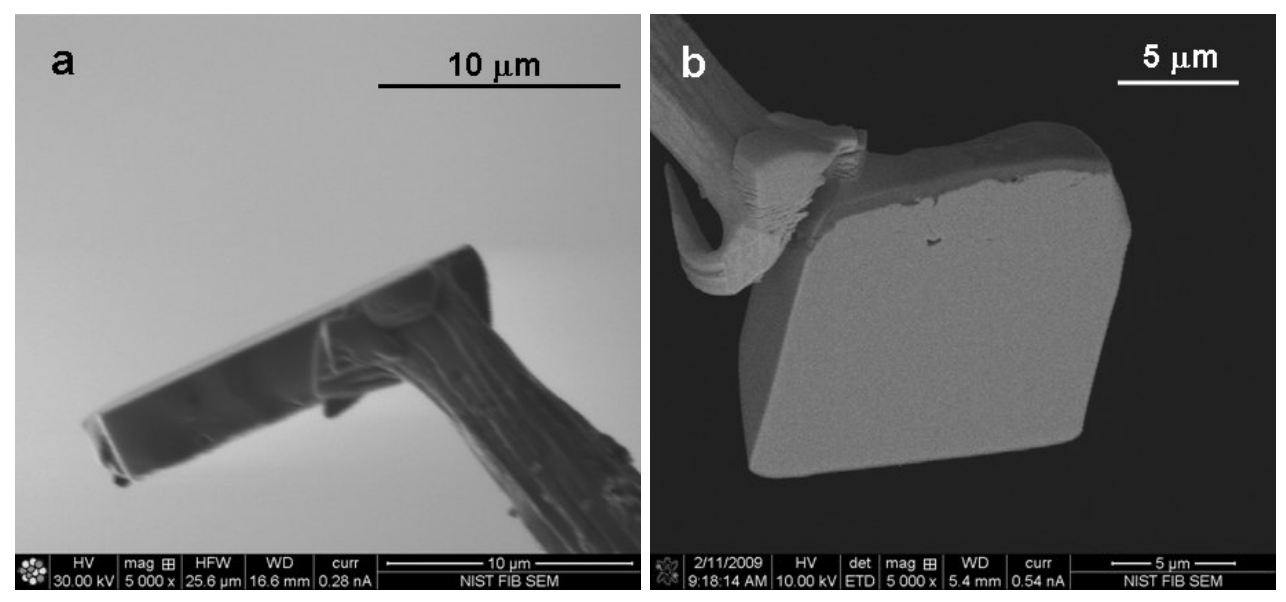

FIG. 1. Ion (a) and electron (b) beam images of a sample volume prepared and oriented for $3 \mathrm{D}$ spectrum imaging while suspended in vacuum. The volume is pre-tilted at $52^{\circ}$ and rotated by $20^{\circ}$ for better access to the EDS detector.
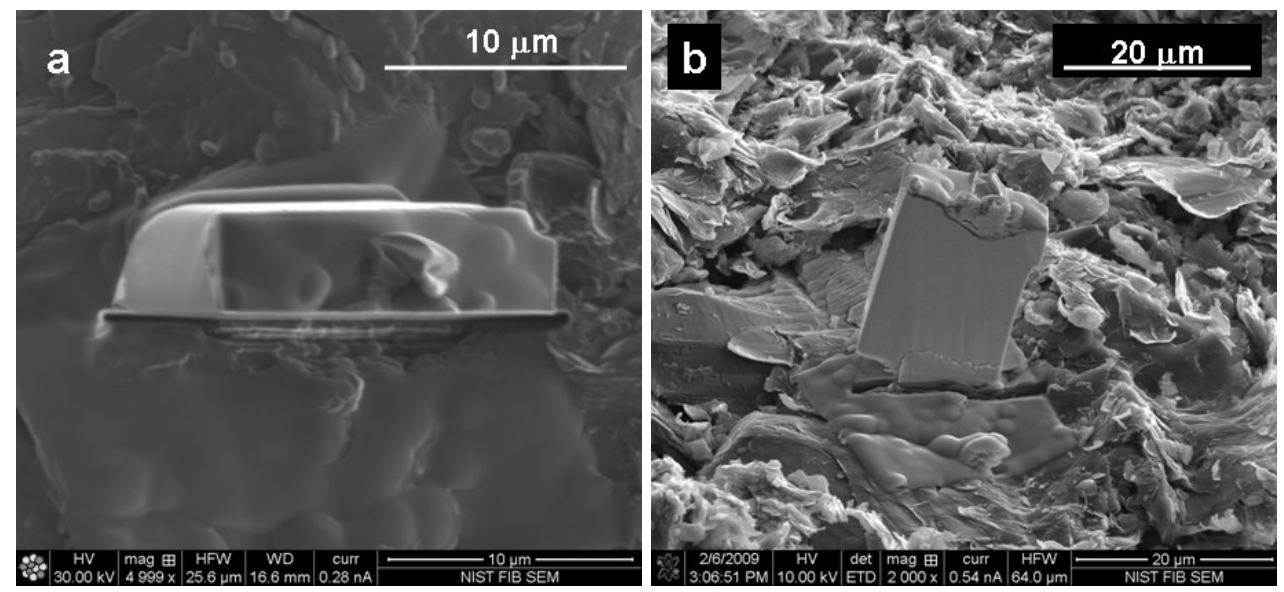

FIG. 2. Ion (a) and electron (b) beam images of a sample volume prepared and oriented for $3 \mathrm{D}$ spectrum imaging on a carbon planchet. 